\title{
Umkämpfte Zugänge zur Bedeutung des Rechts: Die interkulturelle Auslegung in der Verfassungsrechtsprechung Ecuadors und Kolumbiens
}

\author{
Andreas Gutmann
}

Abstract: Zugang zum Recht muss mehr bedeuten, als die Möglichkeit, gerichtliche Verfahren anzustrengen. Soll das Recht tatsächlich zugänglich sein muss es auch die Teilhabe an seiner Ausdeutung ermöglichen. Die Verfassungsgerichte Ecuadors und Kolumbiens haben vor diesem Hintergrund das Werkzeug der interkulturellen Auslegung entwickelt. Diese erfordert es, indigenes Rechtdenken und dessen Eigenlogik anzuerkennen. Intendiert ist hierbei eine Kollision auf Augenhöhe bei der sich die beteiligten Episteme durch einen ordre-public-Vorbehalt gegen einen indifferenten Kulturrelativismus absichern, der durch eine unkritische Annahme fremder Vorstellungen eigene grundlegende Werte zur Disposition stellt. Eine interkulturelle Auslegung erfordert also einen stetigen Übersetzungsvorgang, nicht nur auf sprachlicher, sondern vor allem auch auf epistemologischer Ebene. Sie öfnet einen - nicht immer konfliktfreien - Raum für die Aushandlung rechtlicher Bedeutung.

Der Zugang zum Recht wirkt normierend. Bei der Übersetzung gesellschaftlicher Konflikte in die Sprache des Rechts tritt stets eine Verfremdung ein. ${ }^{1}$ Dies gilt insbesondere auch in Verfahren zur Geltendmachung verfassungsrechtlich garantierter (Grund-)Rechte. Auch wenn diese abstrakt gefasst sind, liegt ihnen doch stets eine bestimmte Vorstellung des rechtlichen Subjekts zugrunde, die nicht auf alle Subjekte gleichermaßen zutrifft. So weist etwa die feministische Rechtswissenschaft seit längerem zutreffend darauf hin, dass das Recht von einem heterosexuellen cis-männlichen Subjekt ausgeht. ${ }^{2}$

Dieser Beitrag beleuchtet ein Instrument, welches das Recht auch für subalterne Vorstellungen und Forderungen öffnen möchte. Das insbeson-

1 Hierzu etwa Teubner/Zumbansen, Zeitschrift für Rechtssoziologie 21 (2000), 189-216.

2 Ritzi, Die Grenzen der Gleichheit: Feministische Kritik am Begriff der Person, in: Gröschner/Kirste/Lembcke (Hrsg.), Person und Rechtsperson, 2015, S. 274 (277); vgl. Brown, Die Paradoxien der Rechte ertragen, in: Menke/Raimondi (Hrsg.), Die Revolution der Menschenrechte, 2011, S. 454 (457). 
dere von der ecuadorianischen und kolumbianischen Verfassungsrechtsprechung entwickelte Werkzeug der interkulturellen Auslegung (interpretación intercultural) eröffnet insbesondere indigenen Gemeinschaften einen Zugang zum Recht, indem es den Auslegungsprozess radikal pluralisiert.

\section{Auslegung als wechselseitige Übersetzung}

Um Forderungen als Rechtssubjekt zu artikulieren, müssen, wie Daniel Loick ausführt, „[a]lle Subjektanteile, die nicht mit der abstrakten Persönlichkeit vereinbar sind [...] überwunden, untergeordnet oder verdrängt werden “. ${ }^{3}$ Dieser Anpassungsprozess wird komplizierter, je größer die Differenz zwischen dem sich artikulierenden und dem hegemonialen, in das Recht eingeschriebenen Subjekt ist. ${ }^{4}$ Häufig wird das Recht von subalternen Gruppen daher nicht als ermächtigend, sondern als Instrument der Herrschaftsausübung erlebt, wie aus einem andinen indigenen Blickwinkel etwa Fernando Huanacuni beschreibt. ${ }^{5}$

Gleichzeitig ist gerade für solche subalternen Gruppen der Zugang zum Recht von großer strategischer Bedeutung, was eine ambivalente Haltung zum Recht hervorbringt. Beispielhaft zeigt sich dies etwa im Kampf um (indigene) Landrechte. Das Verhältnis indigener Gemeinschaften zu dem von ihnen bewohnten und bearbeiteten Land passt häufig nicht zur Vorstellung individuellem Grundeigentums. ${ }^{6}$ Die Forderung nach indigenen Landrechten versucht daher einerseits, Nutzungsweisen als eigentumsähnlich darzustellen, andererseits aber auch neue, etwa kollektive Formen des Grundeigentums im Recht zu etablieren. ${ }^{7}$ Ähnliches zeigt sich im Verhält-

3 Loick, Juridismus: Konturen einer kritischen Theorie des Rechts, 2017, S. 123; ähnl. auch Brown, Paradoxien (Fn. 2), S. 458, die von der „reglementierende[n] Dimension von Rechten" spricht.

4 Corte Constitucional de Colombia, Urt. v. 4.12.2009, Rs. T-903/09, Rn. II. 4.3.

5 Huanacuni Mamami, Vivir bien/Buen Vivir, 6. Auflage, 2015, Ebook Position 2727.

6 Masapanta Gallegos, El derecho indígena en el contexto constitucional ecuatoriano: entre la exigibilidad de derechos y el reconocimiento del pluralismo jurídico, in: Espinosa Gallegos-Anda/Caicedo Tapia/Avila Linzán (Hrsg.), Derechos ancestrales, 2009, S. 409 (433 f.); Corte Constitucional del Ecuador, Urt. v. 18.3.2010, Rs. 001-10-SIN-CC, S. 81 (Sondervotum Nina Pacari); Schacherreiter, Das Landeigentum als Legal Transplant in Mexiko: Rechtsvergleichende Analysen unter Einbezug postkolonialer Perspektiven, 2014, S. 253 f.; von Redecker, Revolution für das Leben: Philosophie der neuen Protestformen, 2020, S. 269-274.

7 Vgl. hierzu etwa Bhandar, Colonial lives of property: Law, land, and racial regimes of ownership, 2018, S. 193-200. 
nis der LGBTIQ*-Bewegung zur Ehe: Diese wird einerseits als patriarchales Institut kritisiert, andererseits wird aber auf deren Öffnung hingearbeitet. ${ }^{8}$

Die Arbeit mit dem Recht kann dabei als wechselseitiger Übersetzungsvorgang beschrieben werden. Für ihre rechtliche Verhandelbarkeit müssen soziale Konflikte in die Sprache des Rechts übersetzt werden. Gleichzeitig muss die abstrakte Sprache des Rechts einen Übersetzungsvorgang durchlaufen, um auf den konkreten Fall angewendet werden zu können. Das Ergebnis dieser Übersetzungsvorgänge ist nicht nur kontingent, sondern auch Ausdruck konkreter historischer Machtverhältnisse.

\section{Auslegung als Hervorbringung von Rechtsbedeutung}

Gleichzeitig bringt die Übersetzung, dies hat etwa Walter Benjamin gezeigt, selbst Bedeutung hervor: „[I]n seinem [des Originals] Fortleben, das so nicht heißen dürfte, wenn es nicht Wandlung und Erneuerung des Lebendigen wäre, ändert sich das Original. Es gibt eine Nachreife auch der festgelegten Worte“.9 Auf das Recht gewendet: Jede Arbeit mit einem Rechtstext wirkt auf dessen Bedeutung zurück.

Wenn Rechtsauslegung selbst Rechtsbedeutung hervorbringt, ${ }^{10}$ muss sich die Zugänglichkeit des Rechts auch am Zugang zur Rechtsinterpretation messen lassen. Soll das Recht tatsächlich zugänglich sein - so die These dieses Beitrages - muss es auch die Teilhabe an seiner Ausdeutung ermöglichen.

In diese Richtung weisen verschiedene Entwicklungen. Das Institut des amicus curiae bietet die Möglichkeit, weitere Stimmen in den Gerichtsprozess zu integrieren. ${ }^{11}$ Strategische Prozessführung versucht bewusst, die Grenzen des Rechts durch die Artikulation neuer Forderungen zu verschieben. Offensive Strategien der Nebenklagevertretung verändern den Fokus eines Strafverfahrens. ${ }^{12}$ Im Folgenden soll jedoch mit der

8 Aus der Rechtsprechung siehe hierzu etwa das Sondervotum von Fernando Cruz Castro in einem Verfahren zur Ehe für alle Sala Constitucional de la Corte Suprema de Justicia, Costa Rica, Urt. v. 8.8.2018, Rs. Res. № 2018012782, Rn. XIII b, das sich diesem Spannungsverhältnis widmet.

9 Benjamin, Charles Baudelaire Tableaux Parisiennes, Vorwort: Die Aufgabe des Übersetzers, in: Gesammelte Schriften Band IV-1, 4. Auflage 2006, S. 9 (12).

10 Ähnl. auch Müller/Christensen, Grundlegung für die Arbeitsmethoden der Rechtspraxis, 2013, S. 283: „Die Rechtsnorm muß vielmehr [durch Auslegung] überhaupt erst gemacht werden."

11 Hierzu Blanquett/Casser, KJ 50 (2017), 94.

12 Siehe etwa Pietrzyk/Hoffmann, KJ 53 (2020), 311. 
insbesondere von den Verfassungsgerichten Ecuadors und Kolumbiens vorgenommenen interkulturellen Auslegung (interpretación intercultural) ein Werkzeug im Fokus stehen, welches den Zugang zum Recht in besonders radikaler Weise öffnet. Die interpretación intercultural ergänzt die überkommenen Auslegungsmethoden und verlangt die Berücksichtigung indigenen Denkens und einen Pluralismus der Interpretationen in einem interkulturellen Dialog über rechtliche Bedeutung. Sie erkennt damit an, dass die Auslegung von Rechtstexten stets auf kulturellen Vorannahmen beruht und fordert gerade die Vielzahl kulturell vorgeprägter Auslegungsmöglichkeiten als fruchtbar wahrzunehmen. Die interpretación intercultural öffnet die Auslegung für den „Kampf[...] ums Recht im Recht durch Recht über das Recht hinaus" ${ }^{13}$ und erkennt an, dass die Bedeutung, die eine (Rechts-)Kultur einem Begriff zuordnet, nicht nur kontingent, sondern selbst das Ergebnis von Aushandlungsprozessen ist.

\section{Hintergrund: Rechtspluralismus und Neokonstitutionalismus}

Die Rechtsordnungen Ecuadors und Kolumbiens sind auch heute von der Kolonialgeschichte geprägt. Auch wenn die häufig kolportierte Behauptung, die Rechtssysteme Lateinamerikas seien als Kopien der europäischen Vorbilder den europäischen Rechtskreisen zugehörig14 in dieser Form jedenfalls verkürzend ist, ${ }^{15}$ kann nicht geleugnet werden, dass sich insbesondere die politischen und wirtschaftlichen Eliten auch nach der formellen Unabhängigkeit von den Kolonialmächten weiterhin nach Europa orientierten. Im Rahmen der verfassungsrechtlichen Strömung des lateinamerikanischen Neokonstitutionalismus entfernten sich zahlreiche innovative Verfassungsgebungen in den letzten Jahrzehnten allerdings immer weiter von dieser europäischen Provenienz. Als erstes neokonstitutionelles Dokument wird häufig die sich bis heute in Kraft befindliche kolumbianische Verfassung von 1991 bezeichnet, ${ }^{16}$ die ecuadorianische Verfassung von 2008 ist hingegen einer der jüngsten entsprechenden Rechtsakte.

13 Fischer-Lescano, Regenbogenrecht: Transnationales Recht aus den „Quellen des Judentums", in: König/Seichter (Hrsg.), Menschenrechte. Demokratie. Geschichte: Transdisziplinäre Herausforderungen an die Pädagogik, 2014, S. 163 (170).

14 Siehe etwa Zweigert/Kötz, Einführung in die Rechtsvergleichung, 3. Auflage, 1996, S. 113.

15 Schacherreiter, Landeigentum (Fn. 6), S. 50.

16 Etwa Noguera Fernández, Revista Vasca de Administración Pública 83 (2009), 117, S. 119. 
Ein wichtiges Charakteristikum des lateinamerikanischen Neokonstitutionalismus ist dessen Bekenntnis zu einem Rechtspluralismus, wie es sich ausdrücklich sowohl in Kolumbien als auch in Ecuador findet. Beide Verfassungen bejahen „die Frage, ob man in einer staatlich organisierten Gesellschaftsordnung davon ausgehen kann, daß es mehr als eine, den Begriff Recht verdienende normative und institutionelle Ordnung oder Teilordnung geben könne“, welche Franz von Benda-Beckmann als „[i]m Zentrum der neuen Diskussionen über Rechtspluralismus" stehend identifiziert. ${ }^{17}$ In beiden Ländern werden indigene Rechtssysteme als eigenständige Ordnungen anerkannt, indigenes und staatliches Recht besteht nebeneinander. Insbesondere Konflikte zwischen Mitgliedern einer indigenen Gemeinschaft und solche, die sich auf Vorkommnisse auf indigenen Territorien beziehen, können nach dem Recht dieser Gemeinschaften geschlichtet werden.

Das Institut der interpretación intercultural wurzelt in diesem Rechtspluralismus, geht aber gleichzeitig darüber hinaus. ${ }^{18}$ Es wurde ursprünglich für Fälle der Kollision zwischen indigenem und staatlichem Recht entwickelt. Strittig ist hier zum einen häufig die Abgrenzung des Anwendungsbereichs staatlicher und indigener Jurisdiktionsgewalt. Diesbezüglich hatte das kolumbianische Verfassungsgericht schon früh festgestellt, dass diese Abgrenzung nicht streng formal stattfinden kann, sondern das „Prinzip der ethnischen und kulturellen Diversität“ berücksichtigen muss. ${ }^{19}$ Zum anderen kann die materielle Vereinbarkeit indigener Justizakte mit dem staatlichen (Verfassungs-)Recht Fragen aufwerfen. ${ }^{20}$ Diese Kollisionslagen dürfen, soll der Rechtspluralismus ernstgenommen werden, nicht stets einseitig zugunsten des staatlichen Rechts aufgelöst werden. Dass nichtstaatliche Rechtsordnungen zu vom staatlichen Recht abweichenden Ergebnissen kommen können, ist gerade das erklärte Ziel des Rechtspluralismus. Gleichzeitig ist die staatliche Rechtsordnung nicht gewillt, jedes beliebige Resultat indigenen Rechts anzuerkennen, sondern zieht gewisse Trennlinien - Andreas Fischer-Lescano spricht von einem ord-

17 Benda-Beckmann, Zeitschrift für Ethnologie 119 (1994), 1 (2).

18 Masapanta Gallegos, Interpretación intercultural desde la jurisprudencia constitucional ecuatoriana, in: Storini (Hrsg.), Constitucionalismo y nuevos saberes jurídicos, 2017, S. 255 (263 f.).

19 Corte Constitucional de Colombia, Urt. v. 26.9.1996, Rs. T-496/96, Rn. 2.4.3.

20 Vgl. Masapanta Gallegos, Derecho indígena (Fn. 6), S. 410, der den Fokus der Debatte über indigene Justiz auf diesen Aspekt kritisiert. 
re public-Vorbehalt ${ }^{21}$ - ein. Rechtspluralismus benötigt also ein Kollisionsrecht, das bei Konflikten zwischen den Rechtsordnungen vermittelt.

\section{Verhandlungen im Zwischenraum}

Dieses Kollisionsrecht kann als Zwischenraum konzeptualisiert werden, in welchem Inhalt und Bedeutung des Rechts ausgehandelt werden. Als Zwischenraum bezeichnet der postkoloniale Denker Homi K. Bhabha ein Dazwischen der Kulturen, in dem in einem Akt der Verhandlung und der Übersetzung 22 ein „heterogenes Gemisch“ oder eine „vermischte Unauflöslichkeit" entsteht. ${ }^{23}$ Gerade das "inter“" ist dabei nach Bhabha „das Entscheidende am Übersetzen und Verhandeln, am Raum da-zwischen", es trage "den Hauptanteil kultureller Bedeutung in sich“. ${ }^{24}$ Durch die Prozesse der Hybridisierung bildet sich hierbei etwas Neues, welches das Vorhergehende jedoch nicht auflöst, sondern reflektiert und gleichzeitig

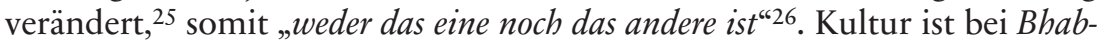
$h a$ stets hybrid und durch Aushandlung und Übersetzung hervorgebracht. Zwischenräume können emanzipatorisch genutzt werden: „Im Prozeß der Übersetzung wird ein weiterer politischer und kultureller Kampfplatz im Zentrum der kolonialen Repräsentation aufgeschlossen. “27 Das Konzept des Zwischenraums erkennt an, dass gerade auch subalternen Positionen Handlungs- und Äußerungsmacht zukommen kann.

Verhandlungen im Zwischenraum finden jedoch nicht lediglich beim Aufeinandertreffen verschiedener Rechtsordnungen statt. So ist auch die einzelne Rechtsordnung von verschiedenen konfligierenden Einflüssen geprägt, ${ }^{28}$ sie ,ist pluralistisch, voller Widersprüche und rechtlicher Inter-

21 Fischer-Lescano, Merkur 74 (2020), 22, 27; von einen „orden público" spricht auch Corte Constitucional de Colombia, Urt. v. 30.5.1994, Rs. T-254/94, Rn. 7.3.

22 Bhabha, Die Verortung der Kultur, 2011, S. 58.

23 So die Bezeichnungen bei Struve, Zur Aktualität von Homi K. Bhabha: Einleitung in sein Werk, 2013, S. 101.

24 Bhabha, Verortung (Fn. 22), S. 58.

25 Hierzu auch Foljanty, Legal Transfers as Processes of Cultural Translation: On the Consequences of a Metaphor, Max Planck Institute for European Legal History research paper series, 2014, S. 12.

26 Bhabha, Verortung (Fn. 22), S. 38.

27 Ebd., S. 51.

28 Nach Álvarez Lugo, Foro: Revista de Derecho 2020, 7 (11) herrscht auch in indigenen Gemeinschaften selbst Rechtspluralismus. 
essengegensätze“ ${ }^{29}$ und darf nicht „als kohärente oder gar eindeutige Ordnung begriffen werden. " 30 Dies gilt in besonderer Weise bei den hier betrachteten Rechtsordnungen. Die Verfassungen Ecuadors und Kolumbiens rezipieren in weitem Umfang europäisches Recht, streben aber gleichzeitig auch eine umfassende Dekolonialisierung durch einen Bruch mit diesen westlichen Einflüssen an. ${ }^{31}$ Besonders deutlich, wird dies, wenn sich die ecuadorianische Verfassung auf indigene Konzepte wie das sumak kawsay ${ }^{32}$ oder die Pacha Mama ${ }^{33}$ bezieht. Die Verfassungen können also selbst als hybrid, als Ergebnis eines interkulturellen Dialogs im Zwischenraum gesehen werden.

\section{Die interpretación intercultural als Auslegungsmethode}

Die interpretación intercultural ist ein offensives Bekenntnis zu diesem internen Pluralismus des Rechts. Insbesondere die ecuadorianische Rechtsprechung hat den Anwendungsbereich der Methode so ausgeweitet, dass sie als neuer Bestandteil des Auslegungskanons potenziell für jede Rechtsfrage herangezogen werden kann, selbst wenn indigene Gemeinschaften nicht direkt betroffen sind. ${ }^{34}$ Hierbei verlangt die interpretación intercultural, dass rechtliche Begriffe auch im Lichte der Vorstellungen der indigenen Gemeinschaften ausgelegt werden. ${ }^{35}$ Sie gründet auf der Erkenntnis, dass der verfassungsrechtlich garantierte Rechtspluralismus nicht nur die Rechtssetzung, sondern auch die Auslegung umfassen muss. ${ }^{36}$ Indem sie innerhalb

29 Fischer-Lescano, Regenbogenrecht (Fn. 13), S. 174.

30 Seinecke, Das Recht des Rechtspluralismus, 2015, S. 293.

31 Nach Pichl, KJ 45 (2012), 131 (139) stellt dies ein allgemeines Charakteristikum postkolonialer Verfassungen dar.

32 Hierzu etwa Acosta, Juridikum 2009, 219. Das Konzept speist sich unter anderem aus indigenen Kosmovisionen des Anden- und Amazonasraums und strebt ein nachhaltiges Lebens- und Entwicklungsmodell an.

33 Siehe hierzu Gutmann, ZUR 2019, 611. Mit Pacha Mama wird eine holistische Wahrnehmung der nichtmenschlichen Umwelt adressiert, die auf Beziehungen und Verantwortlichkeiten zwischen Mensch und Natur beruht.

34 So wohl Corte Constitucional del Ecuador, Urt. v. 9.12.2009, Rs. N. 008-09-SANCC, S, 53; Sala Multicompetente de la Corte Provincial de Pastaza, Urt. v. 11.7.2019, Rs. 16171-2019-00001, S, 20; Masapanta Gallegos, Interpretación intercultural (Fn. 18) S. 263-272.

35 Sala Multicompetente de la Corte Provincial de Pastaza, Urt. v. 11.7.2019, Rs. 16171-2019-00001, S. 19.

36 So für Bolivien Arias López, Anuario Iberoamericano de Justicia Constitucional 18 (2014), 45, 58. 
der staatlichen Rechtsprechung vonstattengeht, ist sie weitreichender als ein Rechtspluralismus, der kollisionsrechtlich das Nebeneinander verschiedener Rechtsordnungen organisiert. ${ }^{37}$ Die interpretación intercultural geht vielmehr von einem Binnenpluralismus innerhalb des staatlichen Rechts und seinen Institutionen aus.

Die Notwendigkeit der interpretación intercultural kann auf verschiedene Weise begründet werden. Sie findet ihren Ursprung etwa in dem Recht indigener Gruppen auf kulturelle Selbstbestimmung ${ }^{38}$ oder im ecuadorianischen Fall in der ausdrücklichen Proklamierung des plurinationalen und interkulturellen Staates. ${ }^{39}$ Dem Prinzip der interculturalidad kommt insbesondere in der ecuadorianischen Verfassung ein hoher Stellenwert zu. Bei diesem handelt es - anders als bei der Multikulturalität - um ein normatives Konzept, das über die Anerkennung der Koexistenz kultureller Vielfalt hinausgeht. ${ }^{40}$ Unter dem von der Indigenenbewegung in den politischen und rechtlichen Diskurs eingebrachten ${ }^{41}$ Begriff der Interkulturalität wird Dialog und gegenseitige Anerkennung zwischen den Kulturen, sowie ein stetiges Lernen vom anderen verstanden. ${ }^{42}$ Es geht gerade nicht darum bestehende Differenzen zu überwinden, sondern diese zu verhandeln und Räume der Begegnung zu schaffen. ${ }^{43}$ Die interpretación intercultural sieht daher den Streit um die Bedeutung des Rechts selbst als wertvoll an. Sie tritt der Vorstellung entgegen, dass (rechtliche) Bedeutung etwas Feststehendes, die Rechtauslegung also als mechanischer, unpolitischer Vorgang zu begreifen ist.

Eine interpretación intercultural ermöglicht hierbei, indigenes Rechtdenken und dessen Eigenlogik anzuerkennen. ${ }^{44}$ Angestrebt ist ein von gegen-

37 Masapanta Gallegos, Derecho indígena (Fn. 6), S. 263 f.

38 Sala Multicompetente de la Corte Provincial de Pastaza, Urt. v. 11.7.2019, Rs. 16171-2019-00001, S. 32.

39 Corte Constitucional del Ecuador, Urt. v. 18.3.2010, Rs. 001-10-SIN-CC, S, 44.

40 Altmann, Die Indigenenbewegung in Ecuador: Diskurs und Dekolonialität, 2014, S. 250; Walsh, Interculturalidad, estado, sociedad: Luchas (de) coloniales de nuestra época, 2009, S. 41.

41 Andrade, Antropología. Cuadernos de Investigación 8 (2009), 13, 22; zur Genealogie Altmann, Indigenenbewegung (Fn.40), S, 246-258; Walsh, Interculturalidad crítica y educación intercultural, in: Viaña Uzieda/Tapia Mealla/Walsh (Hrsg.), Construyendo interculturalidad crítica, 2010, S. 75 (79-88).

42 Andrade, Antropología. Cuadernos de Investigación 8 (2009), 13 (20); Walsh, Interculturalidad (Fn. 40), S. 41.

43 Walsh, Interculturalidad (Fn. 40), S. 45 f.

44 Masapanta Gallegos, Derecho indígena (Fn. 6), S. 424. 
seitigem Respekt geprägter ${ }^{45}$ Dialog auf Augenhöhe. ${ }^{46}$ Es sollen „Räume der Interaktion“ eröffnet werden, die „eine bessere gegenseitige Kenntnis“ ermöglichen. ${ }^{47}$ Dies verlangt von staatlichen Rechtsanwender*innen zunächst, „das ,andere“ zu durchdringen“ und dessen kulturellen Hintergrund verstehen zu versuchen..$^{48}$ Die interpretación intercultural muss sich „dem Wesenszug und den kulturellen Praktiken der betreffenden Gruppen objektiv annähern." ${ }^{49}$ Sollten die verschiedenen Epistemologien in einem Fall zu unterschiedlichen Ergebnissen kommen, muss ein Weg gefunden werden, diesen Konflikt so aufzulösen, dass alle beteiligten Positionen zur weitmöglichsten Entfaltung gelangen. ${ }^{50}$ Die Funktionsweise der interpretación intercultural soll im Folgenden unter drei Gesichtspunkten erörtert werden.

\section{Auslegung als interkultureller Dialog}

Besonders illustrativ ist das Urteil des ecuadorianischen Verfassungsgerichts zur indigenen Universität Amawtay Wasi. ${ }^{51}$ Diese sollte - staatlich akkreditiert - verschiedene auf indigenen Wissensformen basierende Studiengänge anbieten. Ihr Verwaltungssitz befand sich in der Hauptstadt Quito, der Lehrbetrieb sollte an fünf verschiedenen über das Land verteilten Standorten in indigenen Gemeinden stattfinden. Dies untersagte die Bildungsbehörde CONESUP, da nach der geltenden Rechtslage Universitäten erst nach fünf Jahren ihres Bestehens weitere Standorte eröffnen dürften. Der daraus resultierende Rechtsstreit landete schließlich vor dem Verfassungsgericht, das die Gelegenheit nutzte, sich umfänglich zum interkulturellen Charakter der Verfassung und zur Notwendigkeit der interpretación intercultural zu äußern.

45 Sala Multicompetente de la Corte Provincial de Pastaza, Urt. v. 11.7.2019, Rs. 16171-2019-00001, S. 20.

46 Vgl. Corte Constitucional del Ecuador, Urt. v. 9.12.2009, Rs. N. 008-09-SAN-CC, S. 52 .

47 Sala Multicompetente de la Corte Provincial de Pastaza, Urt. v. 11.7.2019, Rs. 16171-2019-00001, S, 32.

48 Corte Constitucional del Ecuador, Urt. v. 9.12.2009, Rs. Nº 008-09-SAN-CC, S. 52.

49 Corte Constitucional del Ecuador, Urt. v. 6.8.2014, Rs. №. 004-14-CN-CC, S. 18.

50 Corte Constitucional del Ecuador, Urt. v. 18.3.2010, Rs. 001-10-SIN-CC, S. 84 (Sondervotum Nina Pacari).

51 Zum Hintergrund siehe Vargas Moreno, Polis 2014, 1. 
Der CONESUP habe es versäumt, sich mit den hinter Amawtay Wasi stehenden indigenen Kosmovisionen zu beschäftigen. Nach diesen könne Wissensweitergabe nicht losgelöst von einem Territorium geschehen, sondern müsse lokal in der indigenen Gemeinde und in deren Beziehung zur nichtmenschlichen Umwelt wurzeln: „[D]as Wissen ist dort in der Natur, in den [indigenen] Völkern selbst, in ihrer Umwelt".52 Dass der CONESUP dies verkannt und das für nicht-indigene Universitäten entwickelte Erfordernis eines festen Hauptsitzes auf Amawtay Wasi angewendet habe, stelle eine verfassungswidrige Diskriminierung dar. ${ }^{53}$ Die Auslegung der entsprechenden Vorschrift durfte also nicht bei den klassischen Auslegungskanones stehenbleiben, sondern musste sie vielmehr vor dem Hintergrund indigener Vorstellungen von Bildung betrachten. Verunmöglicht eine Vorschrift eine solche Bildung, hat sie keinen Anspruch auf eine universelle Geltung, sondern muss sich einem interkulturellen Dialog unterwerfen.

\section{Menschenrechte als Grenze}

Im Amawtay Wasi-Fall kann die Spannung verhältnismäßig leicht aufgelöst werden. Die in einer untergesetzlichen Norm festgehaltene Fünf-Jahresfrist ist nicht Ausfluss von Grundwerten des staatlichen Rechts, ihre Derogation löst keine Krise aus.

Zum Schwur muss es kommen, wenn staatliche und subalterne (Rechts-)Normen derart unterschiedliche Ergebnisse erzielen, dass eine Auflösung nicht mehr ohne weiteres möglich ist. Solche Konflikte werden insbesondere dort vermutet, wo sich indigene Gemeinschaften etwa in Bezug auf Geschlechtergerechtigkeit ${ }^{54}$ oder Formen von Strafe zu den Grund- und Menschenrechten in Widerspruch zu setzen scheinen. Hier liegt die eigentliche Schwierigkeit der interpretación intercultural.

Wie die indigene Verfassungsrichterin Nina Pacari in ihrem Sondervotum zum Urteil des ecuadorianischen Verfassungsgerichts zum Bergbaugesetz (Ley de Minería) ausführt, muss das Recht hier einen Weg zwischen einem Universalismus, der keinerlei Abweichung vom staatlichen Recht zulässt und einem „exzessiven Kulturrelativismus“, der seine eigenen Maß-

52 Corte Constitucional del Ecuador, Urt. v. 9.12.2009, Rs. Nº 008-09-SAN-CC, S. 50 .

53 Ebd., S. 51.

54 Vgl. Schacherreiter, RabelsZ 77 (2013), 272 (276). 
stäbe aufgibt, finden. ${ }^{55}$ Eine Grenze der interpretación intercultural muss - hierüber scheint Einigkeit zu bestehen, anhand der Grund- und Menschenrechte bestimmt werden. ${ }^{56}$ Denn, wie Pacari ausführt: „Eine Gesellschaft ohne Menschenrechte ist blind, aber ohne kulturellen Pluralismus ist sie leer." ${ }^{\text {57 }}$ Gleichzeitig müssen aber auch die Menschenrechte wiederrum interkulturell reflektiert werden. Hierbei öffnet sich ein schwieriges Spannungsfeld. Denn mit Gayatri Chakravorty Spivak gesprochen, sind Menschenrechte einerseits etwas, "you cannot not want", 58 andererseits ist gerade postkoloniale Menschenrechtskritik ${ }^{59}$ durchaus ernst zu nehmen. Diese weist unter anderem darauf hin, dass die Menschenrechte selbst ein westliches Konzept sind, dessen Fokus auf das individuell berechtigte Subjekt ${ }^{60}$ nicht von sämtlichen menschlichen Gemeinschaften geteilt wird $^{61}$ und darüber hinaus die Gefahr einer paternalistischen Unterscheidung zwischen Geber*innen und Empfänger*innen der Menschenrechte besteht. ${ }^{62}$

Deswegen führt das kolumbianische Verfassungsgericht aus, dass die Grund- und Menschenrechte selbst in einem „interkulturellen Konsens“ weitmöglichst" auszulegen seien, und eine Grenze indigener Autonomie erst dann erreicht sein könne, wenn Handlungen vor diesem Hintergrund "wahrhaft intolerabel“ sind. ${ }^{63}$ Die interkulturelle Auslegung ist keine Einbahnstraße, sie wirkt „hin und zurück“ („de ida y vuelta“) ${ }^{64}$ und verändert

55 Corte Constitucional del Ecuador, Urt. v. 9.12.2009, Rs. N. 008-09-SAN-CC, S. 82 (Sondervotum Nina Pacari).

56 Vgl. bereits Corte Constitucional de Colombia, Urt. v. 30.5.1994, Rs. T-254/94, S. 16.

57 Corte Constitucional del Ecuador, Urt. v. 18.3.2010, Rs. 001-10-SIN-CC, S. 82 (Sondervotum Nina Pacari).

58 Spivak, Interviewed by Robert Young: Neocolonialism and the Secret Agent of Knowledge, Oxford Literary Review 13 (1991), 220 (234).

59 Einführend etwa Castro Varela/Dhawan, APuZ 70 (2020), 33.

60 Siehe etwa die Kritik bei Santos, Las paradojas de nuestro tiempo y la Plurinacionalidad, in: Acosta (Hrsg.), Plurinacionalidad: Democracia en la diversidad, 2009, S. 21, 24.

61 So sind nach Brandt/Franco Valdivia, Normas, Valores y Procedimientos en la Justicia Comunitaria, 2007, S. 160 Individualrechte den indigenen Kosmovisionen des Andenraums fremd.

62 Spivak, Righting wrongs: Unrecht richten, 2008.

63 Corte Constitucional de Colombia, Urt. v. 4.12.2009, Rs. T-903/09, S. 21 (Hervorhebung im Original).

64 Villanueva Flores, Revista Derecho del Estado 34 (2015), 289 (301); Sala Multicompetente de la Corte Provincial de Pastaza, Urt. v. 11.7.2019, Rs. 16171-2019-00001, S. 32 . 
die staatliche Verfassung ${ }^{65}$ und die von ihr garantierten Rechte. Im interkulturellen Kollisionsrecht müssen sich die von den beteiligten Rechtsordnungen ausgebildeten ordre-public-Vorbehalte also selbst wieder der Vermittlung durch das Andere unterwerfen.

Eine starre Grenze stellt nach dem kolumbianischen Verfassungsgericht nur der "harte Kern“ der Menschenrechte dar, in allen anderen Fällen müsse die Grenzziehung durch Abwägung vorgenommen werden. ${ }^{66} \mathrm{Im}$ ecuadorianischen Fall ist ein solcher ordre-public Vorbehalt in Art. 171 der Verfassung positiviert, nach dem staatliche Richter*innen Urteile der indigenen Justiz nicht dulden dürfen, wenn diese Menschen- oder Partizipationsrechte $^{67}$ von Frauen mit Verweis auf die Interkulturalität negieren. Auch hier müssen diese Rechte wieder im Lichte der Interkulturalität ausgelegt werden ${ }^{68}$ so dass das Verfassungsgericht - wie es jüngst in Bezug auf interne Regeln einer indigenen Gemeinschaft bezüglich der Zugehörigkeit zu dieser dargelegt hatte - seine Kontrollkompetenz in Bezug auf indigene Gemeinschaften nur in Ausnahmefällen ausübt und dabei „das Prinzip der maximalen Autonomie der indigenen Völker und der minimalen staatlichen Intervention" respektiert. ${ }^{69}$

\section{Wechselseitig reflektierter ordre public}

Die interpretación intercultural stellt demnach einen dialektischen Prozess dar, der "move[s] back and forth between concrete proposals based on existing cultural differences".$^{70}$ Diese Vor- und Zurückbewegung, die nach Arturo Escobar charakteristisch für die Übersetzung ist, hat eine interkulturelle Auslegung zu leiten. So sind alle am Dialog Beteiligten gehalten, ihre fundamentalen Werte in Form eines ordre-public zu formulieren. Diese „Nichtdispositivitäten“ verweisen dabei „auf je unterschiedliche regimespezifische Formulierungen eines Gemeinwohls“ ${ }^{71}$ Die jeweiligen ordrepublic-Vorbehalte werden dann im Anderen reflektiert. So dürfen indi-

65 Masapanta Gallegos, Interpretación intercultural (Fn. 18), S. 272.

66 Corte Constitucional de Colombia, Urt. v. 4.12.2009, Rs. T-903/09, Rn. II. 5.4.

67 Brandt, Indigene Justiz im Konflikt: Konfliktlösungssysteme, Rechtspluralismus und Normenwandel in Peru und Ecuador, 2016, S. 153.

68 Álvarez Lugo, Foro: Revista de Derecho 2020, 7 (16).

69 Corte Constitucional del Ecuador, Urt. v. 9.12.2020, Rs. No. 36-12-IN/2, S. 38.

70 Escobar, Encountering Development: The Making and Unmaking of the Third World, 2. Auflage, 2012, S. 223.

71 Fischer-Lescano, Regenbogenrecht (Fn. 13), S. 177. 
gene Formen der Streitbeilegung nicht vorschnell an vermeintlich feststehenden Parametern eines fair-trial-Grundrechts (debido proceso) gemessen werden, vielmehr müssen die in den jeweiligen indigenen Gemeinschaften bestehenden Vorstelllungen eines fairen Verfahrens in die Ausdeutung des entsprechenden staatlicherseits als orde-public formulierten Grundrechts einfließen. ${ }^{72}$ Die interpretación intercultural sieht sich der Herausforderung ausgesetzt, „die (un)mögliche Übersetzung des Anderen im Eigenen [zu] leisten". ${ }^{73}$

Am Umgang mit devianten Verhalten exemplifiziert: Während das staatliche Recht bei jeglicher Art der Körperstrafe einen Konflikt mit Grund- und Menschenrechten sieht, läuft eine Inhaftierung den Vorstellungen vieler indigener Rechtssysteme entgegen, welche nach Regelverstößen eine Wiederherstellung des durch den Verstoß gestörten Gleichgewichts in der Gemeinschaft anstreben, was durch eine haftbedingte Abwesenheit der rechtsbrüchigen Person vereitelt wird. ${ }^{74}$ Dieser Bruch lässt sich nicht harmonisch auflösen. Er kann jedoch interkulturell reflektiert werden. Funktion und Bedeutung der Nichtdispositivitäten werden selbst Gegenstand der Diskussion.

\section{Eine Herausforderung für das Recht}

Teilweise wird befürchtet, die Anerkennung eines Pluralismus im Recht könne „das moderne, gleiche und freie Recht in vormoderne Ungleichheit und Unfreiheiten zurückzuwerfen". ${ }^{75}$ Dem widersprechen Urteile, welche die interpretación intercultural gerade auf den Grundsatz der Rechtsgleichheit stützen. ${ }^{76}$ Diese verabschieden sich von einem formalistischen Gleichheitsverständnis und statuieren, dass streng schematische Gleichbehandlung durch das Recht diskriminierend wirken kann. Was das bedeutet, zeigt sich in einem kolumbianischen Fall, in dem eine junge indigene Frau für ein politisches Amt kandidiert hatte, ohne das hierfür in einem Dekret vorgesehene Mindestalter von 25 Jahren erreicht zu haben. ${ }^{77}$ In

72 Vgl. hierzu Santos, Las paradojas ( Fn. 60), S. $41 \mathrm{f}$.

73 Fischer-Lescano, Merkur 74 (2020), 22 (30).

74 Vgl. zu diesem Spannungsfeld Corte Constitucional del Ecuador, Urt. v. 6.8.2014, Rs. No. 004-14-CN-CC, S. 28.

75 Seinecke, Rechtspluralismus Fn. 30), S. 13.

76 Corte Constitucional del Ecuador, Urt. v. 6.8.2014, Rs. №. 004-14-CN-CC, S. 30; so auch Masapanta Gallegos, Derecho indígena (Fn. 6), S. 419.

77 Corte Constitucional de Colombia, Urt. v. 27.7.2005, Rs. T-778/05. 
der indigenen Gemeinschaft der Arhuaca, der die Klägerin angehörte, spielt das numerische in Jahren gemessene Alter keine Rolle, die Reife für politische Ämter wird an die körperliche Entwicklung und daran anknüpfende Initiationszeremonien geknüpft. ${ }^{78}$ Würde eine starre, in Jahren bemessene Altersgrenze auch auf das Mitglied der indigenen Gemeinschaft angewendet, würden zwar alle Kandidat"innen formal gleichbehandelt. Gleichzeitig würde die indigene Kandidatin anders behandelt als mögliche der Mehrheitsgesellschaft zugehörige Konkurrent*innen. Deren Reife wird nämlich nach einem in der Mehrheitsgesellschaft, der sie zugehörig sind, verwurzelten Kriterium bestimmt, während der indigenen Kandidatin genau diese Beurteilung nach einem Kriterium, dem in ihrer Gemeinschaft Bedeutung zukommt, versagt würde. Aus diesem Grund, urteilte das Verfassungsgericht, dürfe die betreffende gesetzliche Vorschrift im Falle der Klägerin nicht zur Anwendung kommen. ${ }^{79}$

\section{Die Grenzen der Sprache}

Der Fall verdeutlicht zwei Schwierigkeiten der interpretación intercultural. Zum einen setzt sich das Gericht über den scheinbar eindeutigen Wortlaut hinweg, der prima vista keinen Raum für Auslegung lässt. Es stützt dies nicht ausdrücklich auf die interkulturelle Auslegung, sondern sieht die Norm in einer verfassungskonformen Auslegung als von dem Prinzip der kulturellen Selbstbestimmung verdrängt an und fordert eine „ethno-kulturelle Ausnahme" 80 von der starren Altersgrenze. Auf diese Weise entfällt die Notwendigkeit einer Auseinandersetzung mit der Wortlautgrenze, deren Rigidität auch in der deutschen Rechtsdogmatik Gegenstand von Debatten ist. ${ }^{81}$ In besonderer Weise ist hier zu berücksichtigen, dass sich Sprache dynamisch entwickelt ${ }^{82}$ und somit nicht im machtfreien Raum entsteht, sondern selbst Ausdruck hegemonialer Vorstellungen ist. ${ }^{83}$ Daher

78 Ebd., Rn. II.4.7.

79 Ebd., Rn.. II.7.

80 Ebd., Rn. II.7.1.

81 Hierzu statt vieler Müller/Christensen, Arbeitsmethoden (Fn. 10), S. 313-319 m. w. N.

82 Nach Morlok, Martin/Kölbel, Ralf/Launhardt, Rechtstheorie 31 (2000), 15 (30) können daher „sprachlich verfasste Rechtsnormen ihre Lesart nie determinieren."

83 Illustrativ hierfür etwa die Rechtsprechung. des Schweizer Bundesgerichts zum Frauenstimmrecht. Noch 1957 war es der Meinung, der Begriff „Suisses“ (Schweizer) umfasse eindeutig nur männliche Personen (BGE 83 I 173), erst 1990 kam es 
muss die interpretación intercultural auch hier ansetzen und die Bedeutung von Begriffen selbst infrage stellen.

Indigenes Denken aus dem Andenraum übt häufig Kritik an einem westlichen Logozentrismus. Die Vorstellung, sich einem Ding anhand eines Begriffes zu nähern, stößt hier auf Befremden. Andine indigene Sprachen werden als "verbozentrisch" beschrieben. ${ }^{84}$ Bereits auf semantischer Ebene wird hier also weniger auf eine Ontologie als vielmehr darauf abgestellt, was ein Ding tut und wie es sich in diesem Tun in ein Verhältnis zu seiner Umwelt setzt. Dies ernst zu nehmen, erfordert einen veränderten Umgang mit Rechtstexten. Weniger als der häufig bemühte Blick ins Wörterbuch, ${ }^{85}$ muss hier die Bedeutung der Wahl eines bestimmten Begriffs durch das Recht im Vordergrund stehen. Kann selbst auf diese Weise kein Ergebnis erzielt werden, das dem Recht auf kulturelle Selbstbestimmung und der Berechtigung zur Teilnahme an einem Rechtsdiskurs gerecht wird, kann das Prinzip der verfassungskonformen Auslegung - wie es das kolumbianische Verfassungsgericht exemplifiziert - eine Derogation von der betreffenden Norm erfordern.

\section{Die Gefahr des kulturellen Essentialismus}

Noch schwerer wiegt die Gefahr des kulturellen Essentialismus. Die dichotomische Gegenüberstellung von Mehrheits- und Minderheitskultur kann verdecken, dass diese jeweils uneindeutig, verwoben und verschränkt sind. ${ }^{86}$ In den Worten des kolumbianischen Verfassungsgerichts: „Jede Person ist einzigartig, Gestalterin und Schöpferin ihrer Selbst“ wird aber

zu einem gegenteiligen Ergebnis (BGE 116 Ia 359), siehe hierzu Kramer, Juristische Methodenlehre, 6. Auflage, 2019, S. $137 \mathrm{f}$.

84 Estermann, „Gut Leben" als politische Utopie, in: Fornet-Betancourt (Hrsg.), Gutes Leben als humanisiertes Leben, 2010, S. 261 (265); Oviedo Freire, Ruptura de dos Paradigmas, in: ders. (Hrsg.), Bifurcación del buen vivir y el sumak kawsay, 2014, S. 139 (146).

85 Ausf. zur Beschränktheit dieses Vorgehens für die Rechtsauslegung Müller/Christensen, Arbeitsmethoden (Fn. 10), S.331 f.: „Es kann dem Juristen deshalb nur Plausibilitäten für eine Entscheidung über den Sprachgebrauch an die Hand geben. Es kann ihm diese Entscheidung aber nicht abnehmen." (S. 337 f.).

86 Gerade dies ist nach Walsh, Interculturalidad (Fn. 40), S. 44 eines der Grundanahmen der Interkulturalität; vgl. zu dieser Problematik auch Schacherreiter, RabelsZ 77 (2013), 272 (277). 
gleichzeitig durch „die Art und Weise, wie sie ihr eigenes kulturelles Erbe verinnerlicht, reflektiert und modifiziert" geprägt. ${ }^{87}$

Die interkulturelle Auslegung muss jedoch eine gewisse Deutung eines kulturellen Hintergrundes vornehmen, damit dieser Eingang in das Gerichtsverfahren finden kann. Dies ist zwangsläufig mit einer Festschreibung und Reduktion dessen Bedeutung verbunden. ${ }^{88}$ Die Gerichte verfügen über verschiedene Werkzeuge, um indigene (Rechts-)Vorstellungen im Prozess zu ermitteln. Es ist etwa auf Gutachten von Sachverständigen, wie Soziolog*innen und Anthropolog*innen zurückzugreifen. ${ }^{89}$ Ebenso können Autoritäten (líderes) und andere Mitglieder indigener Gemeinschaften befragt werden, wobei diese Konsultationen, wie der ecuadorianische Provinzgerichtshof in Pastaza betont, selbst barriere- und diskriminierungsarm gestaltet werden müssen. ${ }^{90}$

Auf diese Weise wird durch das Gerichtsverfahren eine gewisse Vorstellung indigener Identität konstruiert und festgeschrieben. Hierdurch manifestieren sich auch in einem inklusiven und interkulturellen Verfahren Machtasymmetrien. So findet die interpretación intercultural vor einem staatlichen Gericht statt und damit in einem Forum, das in einem gewissen Widerspruch zu vielen indigenen Formen der Entscheidungsfindung steht. Wird sich indigenem Wissen - wie oben dargestellt - etwa durch Berichte von Anthropolog*innen genähert, findet dieses vermittelt durch (westliche) wissenschaftliche Praktiken Eingang in den Prozess. Und selbst wenn indigene Gemeinschaften durch Dialogrunden gemeinschaftlich am Rechtsfindungsprozess teilnehmen können, dient dies letztlich dazu, indigenes Wissen so zu verstetigen und zu abstrahieren, dass es verschriftlicht und in ein formalisiertes Verfahren übersetzt werden kann. Gerade die Verschriftlichung von Wissen und dessen Artikulation durch einzelne Expert*innen, stellt in verschiedenen indigenen Epistemologien einen

87 Corte Constitucional de Colombia, Urt. v. 26.9.1996, Rs. T-496/96, Rn. 2.4.2.

88 Etwas ähnliches beobachtet Brown, Paradoxien (Fn. 2), S. 457 in Bezug auf Frauenrechte: „Je deutlicher Rechte als Rechte für Frauen bestimmt sind, [...] desto stärker neigen sie dazu, eine Definition von Frausein, die auf unserer Unterwerfung beruht, im geschichtslosen Diskurs des liberalen Rechtwesens festzuschreiben."

89 Corte Constitucional del Ecuador, Urt. v. 6.8.2014, Rs. №. 004-14-CN-CC, S. 27; Sala Multicompetente de la Corte Provincial de Pastaza, Urt. v. 11.7.2019, Rs.16171-2019-00001, S.31; so auch Masapanta Gallegos, Derecho indígena (Fn. 6), S. 425.

90 Sala Multicompetente de la Corte Provincial de Pastaza, Urt. v. 11.7.2019, Rs.16171-2019-00001, S.31; so auch Arias López, Anuario Iberoamericano de Justicia Constitucional 18 (2014), 45 (57). 
Fremdkörper dar. Wissen wird dort gemeinschaftlich im Tun und in der Beziehung zur nichtmenschlichen Umwelt erlebt und gelebt und zeichnet sich eher durch einen Prozess als durch Hervorbringung gefestigter Bedeutung aus. Diesen Prozess, muss ein Gerichtsverfahren, das zu einem determinierten Zeitpunkt ein feststehendes Ergebnis hervorbringen muss, ${ }^{91}$ unterbrechen.

Diese Erfordernisse des Rechtsprozesses dürfen nach dem ecuadorianischen Verfassungsgericht nicht dazu führen, dass indigene Gruppen im interkulturellen Dialog zu „steinernen Gästen“ („convidados de piedra“) also zu deplatzierten und passiven Teilnehmer*innen des Auslegungsprozesses würden. ${ }^{92}$ Denn hat „das Wissen über den Inhalt einer , anderen' Kultur [...] immer das gute Objekt der Erkenntnis, die geringfügige Summe der Differenz zu sein“, wird nach Bhbabha „eine Beziehung der Herrschaft über sie [reproduziert]". ${ }^{93}$ Soll der interkulturelle Dialog emanzipatorisch sein, muss sich das Recht also öffnen und gewissermaßen selbst zur Disposition stellen. Die geschieht im Rahmen der interpretación intercultural durch eine radikale Pluralisierung des Diskurses über die Funktion rechtlicher Normen. In diesen Diskursen sollen zahlreiche Stimmen, insbesondere aber jene der Betroffenen gehört werden. Wenngleich dies die normierende Wirkung des Rechts nicht überwindet, mildert es sie doch durch die Hinterfragung hegemonialer Sprecher*innenpositionen ab.

\section{Schluss}

In seiner Juridismuskritik sieht Loick in einem „interpretative[n] Pluralismus ${ }^{\text {"94 }}$, den er als zentrales Charakteristikum des jüdischen Rechts identifiziert, einen möglichen Ausweg aus einem Recht, das trennend und normierend wirkt und somit einer gelingenden Sozialität entgegensteht. Als „richtig“ soll nach Loick keine bestimmte Auslegung, sondern „der Streit der Rechtsauslegungen selbst" angesehen werden..$^{95}$ "Motor der Rechtsentwicklung“ ist in einem solchen Rechtsverständnis „Differenz und nicht

91 Nach Derrida, Gesetzeskraft, 1996, S. 54 ist „der Augenblick der Entscheidung [...] stets ein endlicher Augenblick der Dringlichkeit und der Überstürzung".

92 Corte Constitucional del Ecuador, Urt. v. 9.12.2009, Rs. Nº 008-09-SAN-CC, S. 52; so auch Solano Paucay, Revista Killkana Sociales 2 (2018), 33 (36).

93 Bhabha, Verortung (Fn. 22), S. 48.

94 Loick, Juridismus (Fn. 3), S. 320.

95 Ebd., S. 317. 
Konsens“. ${ }^{96}$ Der Zugang zur Rechtsauslegung muss demnach allen Rechtsanwender"innen offenstehen.

Die interpretación intercultural radikalisiert Peter Häberles „offene Gesellschaft der Verfassungsinterpreten“" ${ }^{77}$ Auch in dieser gibt es „keinen Numerus clausus der Verfassungsinterpreten“, „alle Bürger und Gruppen“ nehmen hier am Auslegungsprozess teil..$^{98}$ Gleichsam bleibt Häberle einem gewissen gerichtlichen Dezisionismus verhaftet, indem er von einer allgemeingültigen Festschreibung rechtlicher Bedeutung durch einen zentralisierten Spruchkörper ausgeht. ${ }^{99}$ Den pluralistischen Diskurs um das Recht verortet Häberle „[i]m ,Vorfeld“ juristischer Verfassungsinterpretation der Richter", deren Prüfungsmaßstab gerade dort besonders streng sein solle, wo „in der Öffentlichkeit großer Dissens herrscht. “ 100 Der Streit um rechtliche Bedeutung muss also durch eine autoritative und letztverbindliche Festschreibung eingehegt werden und kann nicht selbst als der Inhalt des Rechts gefasst werden.

Genau hier setzt auch die interpretación intercultural an. Sie hinterfragt die Gewaltförmigkeit der Festschreibung von Bedeutung durch (vermeintlich) feststehende Begriffe. Weniger als die Arbeit mit Begrifflichkeiten steht hier also die Funktion einer rechtlichen Vorschrift für die Gemeinschaft der Rechtsunterworfenen im Vordergrund, weniger als die Dezision die Interpretation als solche. ${ }^{101}$ Zugang zum Recht bedeutet demnach die Möglichkeit der Beteiligung an einem Diskurs im Recht über das Recht.

96 Fischer-Lescano, Regenbogenrecht, (Fn 13), S. 177.

97 Häberle, JZ 30 (1975), 297; eine ausdrücklichen Bezug der interpretación intercultural zu Häberle stellt etwa Arias López, Anuario Iberoamericano de Justicia Constitucional 18 (2014), 45 (57) her.

98 Häberle, JZ 30 (1975), 297.

$99 \mathrm{Zu}$ Recht weist Hailbronner, Der Staat 53 (2014), 425 (426) darauf hin, dass Häberle „einiges bereits voraus[setzt], nämlich die Autorität des Bundesverfassungsgeriechts zur letztverbindlichen Entscheidung über die Ansichten anderer Verfassungsinterpreten."

100 Häberle, JZ 30 (1975), 297 (303), Hervorhebung im Original.

101 Ein solches Verständnis des Rechts entfaltet auch Benjamin, Franz Kafka, in: Gesammelte Schriften Band II 2, 7. Auflage, 2019, S. 409 (437) in seiner Lesart von Kafkas Bucephalos: „Das Recht, das nicht mehr praktiziert und nur studiert wird, das ist die Pforte der Gerechtigkeit.“ 